\title{
Verification of the heat transfer model for screw reactor
}

\author{
Anatoliy Levin*, Alexander Kozlov, Denis Svishchev, and Maxim Penzik \\ Melentiev Energy System Institute SB RAS, 130, Lermontov St., Irkutsk, 664033, Russia
}

\begin{abstract}
At present, the screw reactor pyrolysis is a promising and rapidly developing technology, because it helps obtain the end product, which is charcoal, tar, and synthesis gas. Additionally, pyrolysis can be considered as a preliminary stage for making the charcoal of the preset conversion for its further gasification when obtaining coal-derived gas. This research aims at establishing relationships between the operational parameters of pyrolysis and obtaining of the charcoal of preset conversion by means of mathematical modeling, full-scale experiment, and instrumental studies. Such comprehensive research ensures the accurate initial data, verification of the model, and optimal parameters for obtaining the product with preset properties as well as for further scaling of the reactor. In terms of design the pyrolyzer represents a recuperative heat exchanger where a mix of stack and recirculation gases is considered as a heat carrier. To prevent sintering of particles the screw transportation of fuel is used. The Comsol Multiphysics Software is used as a simulation environment. Heat exchange in the process of pyrolysis is simulated considering physical properties (porosity, permeability, etc.) of the medium. The constructed calculated mesh consisted of 604 thousand elements of three types (tetrahedrons, prisms and pyramids) and had the minimum size of $0.2 \mathrm{~mm}$.
\end{abstract}

\section{Introduction}

According to the International Renewable Energy Agency (IRENA), in 2015 biomass generated $476933 \mathrm{GWh}$ of electricity, which represents $8.6 \%$ of total power generated by renewable energy sources $(5537516 \mathrm{GWh})$. At the same time, the growth of electricity produced by the biomass processing technologies from 2005 (195749 GWh) to 2015 was about $60 \%$ [1]. The main technology for obtaining electricity from biomass is thermochemical conversion. It accounts for about $75 \%$ of the electricity generated. The key reasons for the increase in the use of thermochemical conversion technologies were: increased energy consumption in developing countries, which led to the growth and significant fluctuations in world prices for hydrocarbons; considerations of energy security and general trends towards diversification of energy carriers in the fuel and energy balance; the new concept of distributed generation of energy, suggesting a reduction in the transport range by using local fuels; environmental safety that implies the use of renewable solid fuel resources, including waste $[2,3]$. At present time, 160 projects for the design and construction of stations using the technology of biomass thermochemical conversion are being implemented by the international energy agency in the member countries of the Organization for Economic Co-operation and Development (OECD) [4]. However, the high-quality processing of biomass and waste has been only implemented as part of large installations with a unit capacity of more than $30 \mathrm{MW}$, and their application is unprofitable if extraction and transportation of fuel are costly [5]. To solve the problem of efficient use of such fuels, it is necessary to develop a set of technical solutions aimed at obtaining generator gas of acceptable quality and ensuring the production of electric and thermal energy at sufficiently low cost. The latter condition is the main impediment in resolving this issue in Russia and in other countries [6].

A substantional share of costs of the installed capacity as well as of operating costs of the realized conversion units is related to producer gas purification systems. In this regard, it is necessary to develop either existing gas cleaning systems or design alternative technologies for biomass thermochemical conversion, which by default imply the production of gas of acceptable quality for use in heat engines. One of such technologies is the technology of multi-stage gasification, which, due to the step-by-step organization of the process, allows for production of gas with a minimum content of tar products [7]. The key step of the multistage biomass gasification is pyrolysis, which, as a rule, is organized in a separate screw reactor, since it is pyrolysis that predetermines the further effectiveness of gasification. By varying the operating parameters of the pyrolysis process, one can vary both the composition of the producer gas and the yield of the target product (gas, tar, charcoal) [8]. As noted in [9], for all the universality of the screw reactor, its scaling seems to be a rather challenging task. This is confirmed by the fact that the multi-stage gas generator of the Viking type was scaled only up to $250 \mathrm{~kW}$ [10]. Apparently, the successful use and scaling of the screw pyrolyzer depend on a comprehensive study of the physicochemical properties of the biomass and the operating parameters of the process. It is also important to develop mathematical models that imitate the process under study and predict its progress. This work presents

\footnotetext{
* Corresponding author: lirt@mail.ru
} 
the results of experimental research that was carried out at the laboratory stand of biomass pyrolysis in a screw reactor and provides results of mathematical modeling and verification of the model developed.

\section{Experimental part}

The experimental study was carried out at the laboratory stand of a screw pyrolyzer, the technological scheme of which is given at Fig. 1. The test material was wood pellets with the following charateristics: $6-8 \mathrm{~mm}$ in diameter; $2-5 \mathrm{~cm}$ long; ultimate and proximate analysis: $\mathrm{C}-48 \%, \mathrm{H}-4,6 \%, \mathrm{O}-47,5 \%$; devolatilization $81,7 \%$, ash content $2,2 \%$, moisture $8,2 \%$.

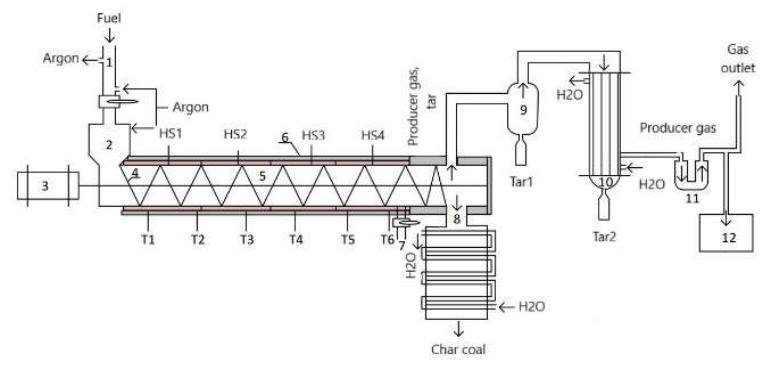

Fig. 1. Technological scheme of the laboratory stand of a screw pyrolyzer: 1 - fuel bunker, 2 - fuel storage tank, 3 screw drive, 4 - screw, 5 - reactor, 6 - thermal isolation, 7 sampler, 8- charcoal storage tank, 9- cyclon, 10 - heat exchanger, 11 - filter, 12 - gas chromatograph, T1-T6 thermocouples, HS1-HS4 - heater sections.

The pellets in the reactor shaft (5) were moved by means of a screw (4) rotated by a variable speed drive (3). In the experiment, three rates of pellet transfer along the length of the reactor were used: $0.19,0.37$, and $0.6 \mathrm{~mm} / \mathrm{s}$. The wood pellets were heated by four sections of the electric heater HS1-HS4 located on the outer surface of the reaction chamber. Figure 2 shows the heating of the pyrolysis reactor at different fuel velocities.

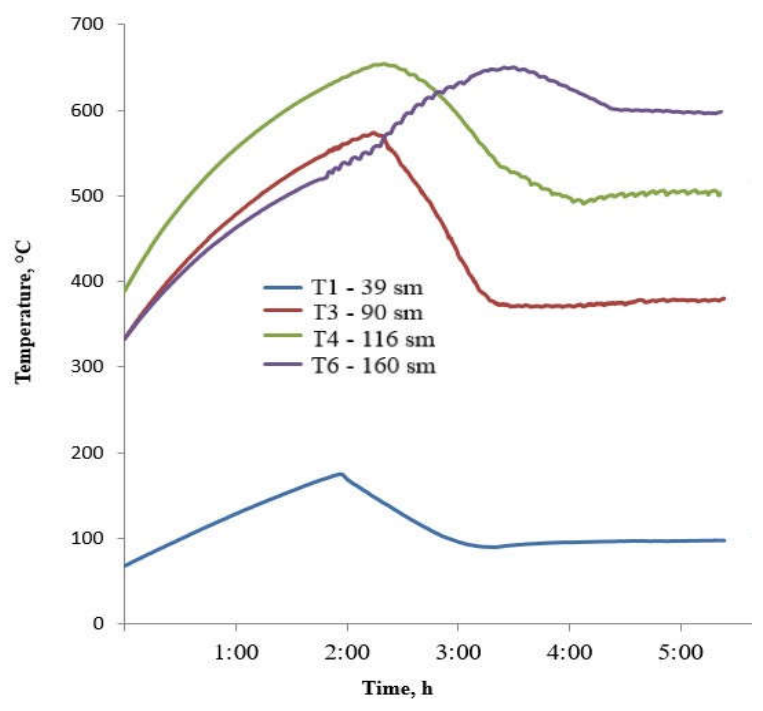

Fig. 2. Heating of the pyrolysis in different sections at the fuel velocity $0.37 \mathrm{~mm} / \mathrm{s}$
It can be seen from Fig. 2 that the initial warm-up stage lasts for two hours, and then we observe a decrease in the temperatures of the sections. It takes the screw pyrolyzer an hour to reach the stationary state.

To estimate the yield of producer gas, we used a balance for the inert gas-marker (argon), a certain amount of which was continuously supplied to the unit.

The hopper was purged with argon to remove air in the fuel mass. Then the fuel enters the storage tank (2) through an airproof gateway. To calculate the pellet consumption, the time of arrival of each portion of fuel into the storage tank was fixed.

The bulk of the reacted wood pellets enters the cooled charcoal storage tank (8). Sampling of charcoal to determine its technical characteristics (moisture, ash content, volatilization, elemental composition, heat of combustion) is carried out through the sampler (7). The producer gas enters the cyclone (9), in which solid particles and high boiling tar (Tar 1) are separated. The producer gas is cooled in the heat exchanger (10), where a low-boiling tar (Tar 2) is separated, and then passes through the filter (11). The yield of liquid products of the wood pyrolysis is estimated by the weight of the tar samples taken from the cyclone (9) and the heat exchanger (10). The thermocouples T1-T6 were used to measure the temperature of the pyroliser wall. The thermocouples that measured the layer temperature were immersed into the layer through special ports in the chamber wall. As a result of the experiment, we obtained the charcoal with a maximum conversion rate of $97 \%$ at the fuel velocity of $0.19 \mathrm{~mm} / \mathrm{s}$. We also drew the diaghram of the change in the bulk density of wood pellets depending on different degrees of conversion (Fig. 3).

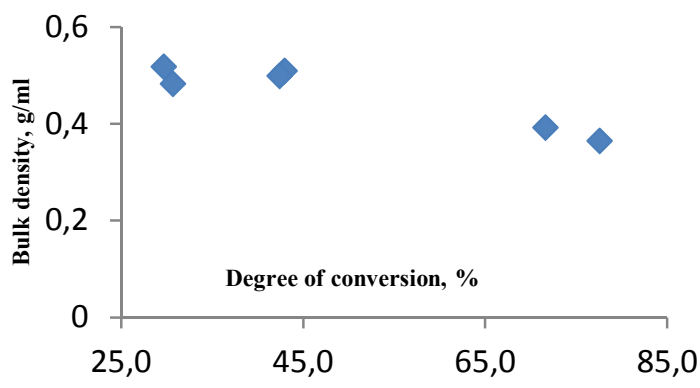

Fig. 3. Change in the bulk density of wood pellets depending on different degrees of pellets conversion.

Figure 3 shows that the bulk density varies insignificantly for samples with a degree of conversion up to $50 \%$. The explanation for this is as follows: when pellets are pyrolyzed to a given degree of conversion, the geometric dimensions of the particles are preserved. It is worth noting that this dependence is further used in the mathematical model describing the pyrolysis process.

\section{Mathematical model}

We used the software based on the corresponding algorithms that ensure good convergence for the differential equations. The calculation mesh includes different number of cells: 
- For gas part it includes hot gas from internal combustion engine and gas inside the screw above the porous medium.

- For a surface of solid elements, it includes all solid surfaces.

- For the others it includes solid volumetric parts such as screw, spiral, coating, etc.

The Comsol Multiphysics Software is used as a simulation environment. Heat exchange in the process of pyrolysis is simulated considering physical properties (porosity, permeability, etc.) of the medium [3]. The equation of a diffusion barrier for the plane separating the porous solid body from the gas space in the pyrolyzer is set as follows:

$$
\begin{aligned}
& -n D_{s, i} \nabla c_{i, u}=\frac{D_{s, i}}{d_{s}}\left(c_{i, u}-c_{i, d}\right) \\
& -n D_{s, i} \nabla c_{i, d}=\frac{D_{s, i}}{d_{s}}\left(c_{i, u}-c_{i, d}\right)
\end{aligned}
$$

where $d_{s}$ - barrier thickness, mm; $D_{s i}-$ diffusion coefficient, $\mathrm{m}^{2} / \mathrm{s}$. In the calculations we assumed a bed thickness of $5 \mathrm{~mm}$ and a diffusion coefficient of $10^{-6} \mathrm{~m}^{2} / \mathrm{s}$. The boundary conditions chosen to solve the problem are:

An inlet temperature of fuel is $20^{\circ} \mathrm{C}$;

An inlet temperature of heating gases is $650^{\circ} \mathrm{C}$;

A velocity of fuel in pyrolyzer $0,2 \div 3 \mathrm{~mm} / \mathrm{s}$;

An inlet velocity of heating gases is $0,5 \mathrm{~m} / \mathrm{s}$.

We calculated the heat required for the water evaporation by determining the concentration of the resulting vapors: $\nabla \cdot\left(-D_{H 20} \nabla c_{H 20}\right)+u \cdot \nabla c_{H 20}=70200 e^{-\frac{34200}{8.314 T}}$. (2)

Conversion of the solid fuel to coke was found using the equation

$$
\nabla \cdot\left(-D_{c o k} \nabla c_{c o k}\right)+u \cdot \nabla c_{c o k}=54500 e^{-\frac{96000}{8.314 T}}
$$

The coefficients of the right-hand side of Eqs. (2), (3) were determined by the thermogravimetric analysis performed on solid fuel samples. It follows from Eq. (3) that the degree of fuel conversion is directly affected only by the temperature distribution, since the movement speed of solid particles is determined only by the rotation speed of the screw.

\section{Analysis and modelling}

As a result of parametric calculations within the abovementioned range for the solid fuel velocity, we obtained distributions of the speed, temperature, and concentration fields of the substances formed. Figure 4 shows an example of spatial distribution of the coke concentration expressed in terms of the maximum achievable value (\%). The spatial inhomogeneity of the conversion can be explained by the essential dependence of the temperature fields on the geometry of the screw, which has a high

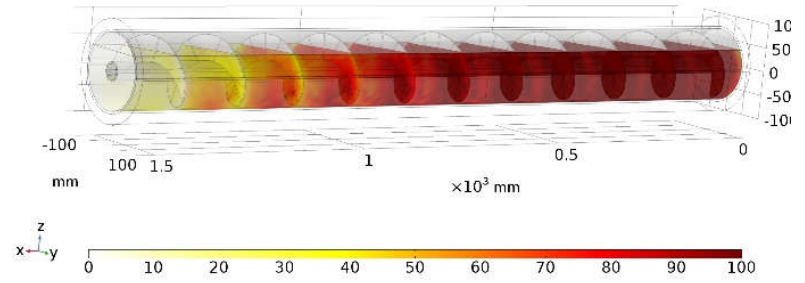

Fig. 4. Spatial distribution of the degree of wood bulk conversion into coke $X_{\text {coke }}[\%]$ thermal conductivity. The influence of the fuel velocity on the solid fuel conversion is illustrated by Figure 5. It can be clearly seen that most of the wood bulk turns into coke halfway through the pyrolyzer.

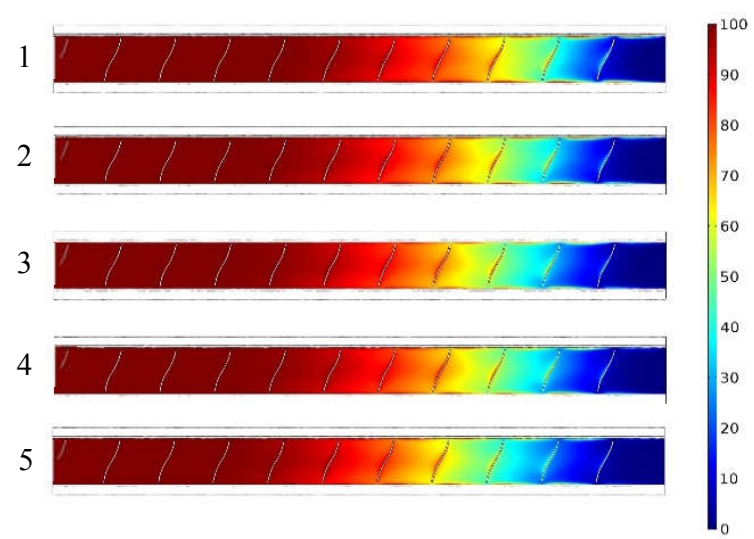

Fig. 5. Degrees of wood bulk conversion into coke for different movement speeds $[\mathrm{mm} /$ hour] $1-106 ; 2-140 ; 3-176 ; 4-212 ; 5-248$.

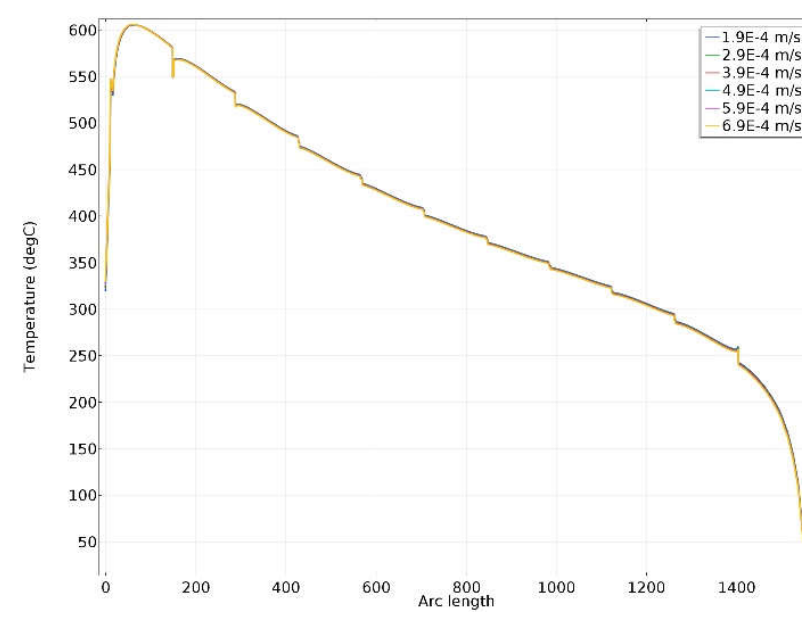

Fig. 6. Temperature distribution along the pyrolyzer for different movement speeds.

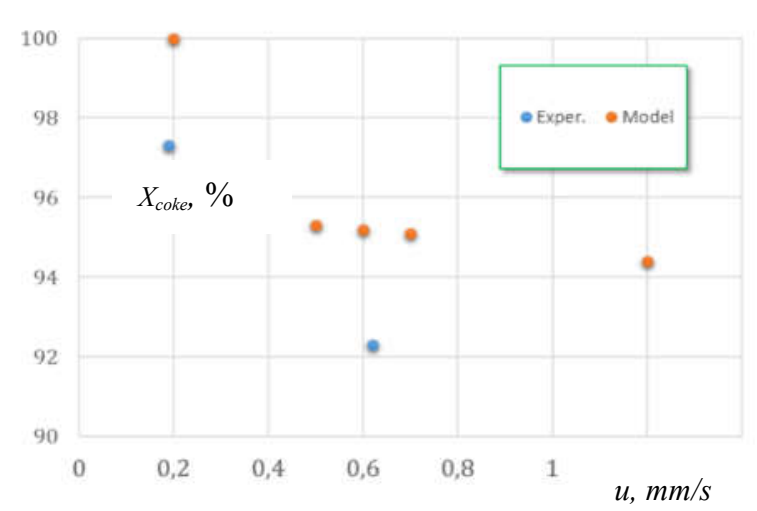

Fig. 7. Comparison between the charcoal conversion degrees obtained experiminally and via numerical modeling.

It can be seen from Figure 6 that the temperature profile is almost the same for all investigated solid fuel flow velocities through the screw reactor. As a result, the relationship between the final fuel conversion in the outlet 
section of the reactor and the rate of the medium should have the following form

$$
X=X_{0} / u \text {, }
$$

where $X_{0}$ is found from the experimental conditions. Equation (3) should hold for $u=0 \div 0,01 \mathrm{~m} / \mathrm{s}$.

Figure 7 shows comparison of the experimental data obtained for different fuel velocities with the caluculated values. We can see a good agreement for the fuel velocities 0.19 and $0,37 \mathrm{~mm} / \mathrm{s}$. If the fuel velocity is high $(0.6 \mathrm{~mm} / \mathrm{s})$, the mathematical model derives the bigger fuel conversion degree than the one obtained experimentally. This can be explained as follows: in contrast to laboratory research, in the experimental facility the solid fuel particles form a close-packed backfill that prevents free convection of gases formed as a result of pyrolysis reactions. As a result, a diffusion barrier arises and limits the reaction rate.

\section{Conclusions}

The model of heat transfer and aerodynamics of the pyrolyzer was developed for express optimization calculations. Inclusion of the kinetic block in the model of heat transfer made it possible to determine the concentration fields of the formed substances (charcoal, steam, etc.).

\section{Acknowledgements}

This research was performed at the Melentiev Energy Systems Institute of SB RAS under support of the Russian Science Foundation (Grant No.16-19-10227) using the High Temperature Circuit Multiple Access Research Center.

\section{References}

1. Brassard P., Godbout S., Raghavan V. Biosystems engineering 161, 80- 92. (2017)

2. Funke A., Henrich E., Dahmen N., Sauer J. Energy Technology, 5(1), 119-129. (2017)

3. http://www.irena.org/bioenergy

4. Molino A., Chianese S., Musmarra D. Journal of Energy Chemistry. 25, 1, 10-25. (2016)

5. M. Castaldi, J. van Deventer, J.M. Lavoie, J. Legrand, A. Nzihou, Y. Pontikes, X. Py, C. Vandecasteele, P.T. Vasuedevan Waste and Biomass Valorization. 8, 6, 1875-1884. (2017).

6. http://www.ieabioenergytask33.org

7. Hupa M., Karlstrom O., Vainio E. Proceedings of the Combustion Institute. 36, 1, 113-134. (2017)

8. A. Kozlov, D. Svishchev, O. Marchenko, S. Solimin, V. Shamansky, A. Keiko // Setting the course for a biobased economy. Proceedings of the 25th European Biomass Conference / Ed. by L. Ek, H. Ehrnrooth, N .Scarlat, A. Grassi, P. Helm. - ETAFlorence Renewable Energies, 776-781. (2017).

9. Heidenreich S., Foscolo P.U. Progress in Energy and Combustion Science. 46, 72-95. (2015).

10. Aramideh S., Xiong Q., Kong S.-Ch., Brown R.C. Fuel. 156. 2. 234-242. (2015)
11. Skov I.R., Mathiesen B.V., Connolly D. A review of biomass gasification technologies in Denmark and Sweden (Department of Development and Planning, Aalborg University, 2013).

12. Levin A.A., Shamansky V.A., Kozlov A.N. Journal of Physics Conference Series, 754, 02206. (2016) 